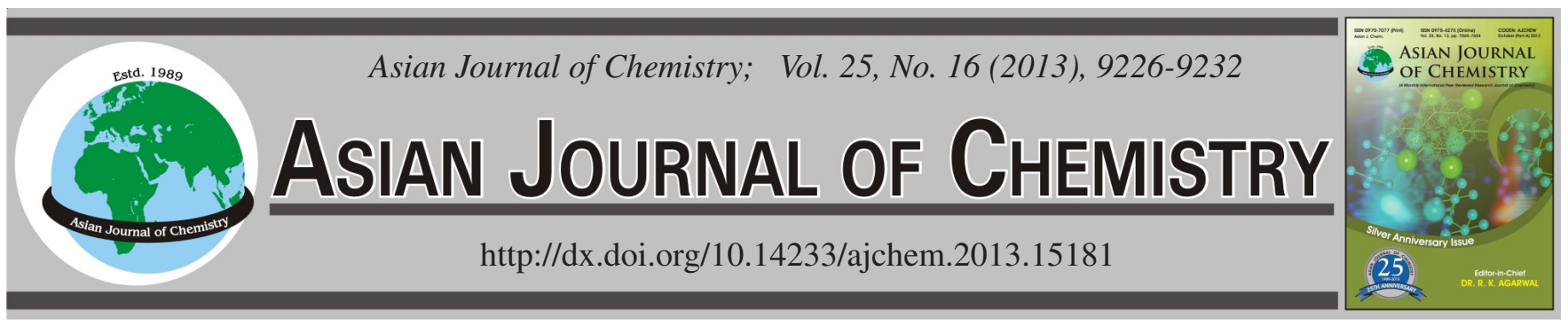

\title{
Distance Dependent Surface Enhanced Raman and Fluorescence by Supported 2D Assembly of Plasmonic Metal Nanoparticles
}

\begin{abstract}
Nur Ahamad ${ }^{1, *}$, MD. Al-Amin ${ }^{1}$ and A. IANOUl ${ }^{2}$
${ }^{1}$ Department of Chemistry, Shahjalal University of Science and Technology, Sylhet-3114, Bangladesh

${ }^{2}$ Department of Chemistry, Carleton University, 1125 Colonel By Dr., Ottawa, ON, Canada

*Corresponding author: Fax: +880 821 715257; Tel: +880 821 713491x251; E-mail: nuruddinahamad@yahoo.com; nur-che@ sust.edu

(Received: 21 January 2013;

Accepted: 20 September 2013)

AJC-14150

Metal nanoparticles polyelectrolytes nanocomposite substrates consisted of variable number of poly(sodium-4-styrenesulfonate)/ poly(diallyldimethylammonium chloride) bilayers deposited on supported 2D assembly of silver nanoparticles were fabricated using layer by layer deposition technique. Rhodamine $6 \mathrm{G}$ and poly(fluorescein isothiocyanate allylamine hydrochloride) were subsequently deposited onto these substrates to investigate distance dependent surface enhance Raman scattering and fluorescence of these substrates. The as prepared substrates exhibited surface enhanced Raman scattering and fluorescence activities. Even though precise distance control between rhodamine $6 \mathrm{G}$ and nanoparticles was rather impossible due to the fluorophore diffusion, a 14 fold enhancement of the rhodamine 6G fluorescence signal was detected. In addition, surface enhanced Raman scattering enhancement of rhodamine 6G containing substrates was noticed at a separation distance corresponding one bilayer. On the contrary for poly(fluorescein isothiocyanate allylamine hydrochloride), a modest 2-fold enhancement of fluorescence was observed for a separation distance corresponds to 30 bilayers.
\end{abstract}

Key Words: Polyelectrolytes, Nanoparticles, Diffusion, Surface enhanced Raman scattering, Surface enhanced fluorescence.

\section{INTRODUCTION}

Metal nanoparticles (NP) and their ordered nanostructures are attracting increasing attention in nanotechnological disciplines due to their unique optical properties arising from the collective oscillation of free electrons in the conduction band upon interaction with external electromagnetic field ${ }^{1-3}$. These optical properties can be tuned by changing size, shape, surrounding dielectric environment as well as interparticles gaps within nanoparticles assembly. Tailoring optical properties of plasmonic nanostructures is important for using them in practical application such as in optics, bionanotechnology and analytical chemistry ${ }^{4,5}$.

It is well known that plasmonic metal nanostructures, exhibiting localized surface plasmon resonances, can be employed to enhance local electromagnetic field which modifies the absorption, scattering and emission processes of near-by dipoles ${ }^{4,6}$. These enhanced local fields are the origin of surface enhanced Raman scattering (SERS) and surface enhanced fluorescence $(\mathrm{SEF})^{7}$. Such optical field enhancing effects are being widely used in Raman and fluorescence based measurements in molecular detection, molecular biology and medical diagnostics ${ }^{8,9}$. Since both SERS and SEF are known to be localized phenomena, therefore understanding lateral as well as axial spatial dependence of the Raman and fluorescence signal enhancement with respect to the enhancing substrates is critical for their optimal use.

Our research group studied the lateral spatial distribution of fluorescence enhancement for a system containing Rhodamine 6G (Rh6G) and a monolayer of silver nanoparticles with polyelectrolyte multilayer as spacer. The important finding of this investigation was the inhomogeneous distribution of fluorescence enhancement in polyelectrolyte-nanoparticle substrate $^{10}$. Therefore it was of further interest to investigate the axial spatial dependence of both Raman and fluorescence enhancement in such polyelectrolyte-nanoparticle substrates.

Distance dependent enhancement of Raman and fluorescence involve investigation of optical signal enhancement of probe molecules at various distances from the metal nanostructures. Various protocols are available for controlling the distance between metal nanostructure and the photosensitive molecules i.e., alternating monolayers of biotinylated bovine serum albumin-avidin, silica coating around the nanoparticles, sequential deposition of oppositely charged polyelectrolyte or various spacer groups having different lengths ${ }^{11-13}$. Layer by layer (LBL) process has evolved as a facile and simple technique for fabrication of ordered nanostructures and thin films for practical purposes ${ }^{14}$. One of the advantages of layer 
by layer technique is the ability to incorporate various materials of interest including dyes, biological agents like proteins, DNA, antibodies etc. ${ }^{15,16}$. Distance control by polyelectrolyte using layer by layer technique provides the control over the thickness of the film ${ }^{17}$.

A reliable investigation of the distance dependence of Raman and fluorescence not only depends on how well the distance is controlled but also on how uniformly the fluorophore molecules are distributed on the surface of the spacer layer. Moreover, interactions between probe molecules and spacer matrix might affect the molecular distribution of the probe molecule on the substrates. Therefore, interaction of photosensitive molecules with the surface of polyelectrolyte film is an important issue to precisely investigate distance dependent nature of SERS and SEF.

In this present work, we engineered nanostructure substrates consisted of silver nanoparticles with polyelectrolyte i.e., [poly(sodium-4-styrenesulfonate) (PSS)/poly(diallyldimethylammonium chloride) (PDADMAC)] layers of varying thickness deposited on the top followed by adsorption of two dyes, Rh6G or poly(fluorescein isothiocyanate allylamine hydrochloride) (PAH-FITC), on the surface of the polyelectrolyte films. Deposition of dyes on the polyelectrolyte films is because of electrostatic interaction between positively charged dye molecules and negatively charged polyelectrolyte surface (outer PSS layer). But hydrophobic interaction, caused by organic moieties, between the polyelectrolytes and the dye molecules could not be ignored. In our present study, Rh6G and PAH-FITC were employed as mobilized and immobilized (polyelectrolyte bound) probes, respectively to study distance dependent nature of SERS and SEF. This study will assist to conduct reliable measurement of fluorescence and Raman based molecular detection.

\section{EXPERIMENTAL}

Poly(diallyldimethylammonium chloride) solution (PDADMAC; 35 wt \% in water, MW < 100,000), poly(sodium- 4-styrenesulfonate) (PSS; MW 70,000), rhodamine 6G (Rh6G), poly(fluorescein isothiocyanate allylamine hydrochloride) (PAH-FITC; MW ca. 6031) and sodium borohydride $98 \%$ were received from Aldrich. The hydrogen peroxide $30 \%$, sodium citrate and silver nitrate were received from Caledon.

Preparation of colloidal metal nanoparticles: A colloidal solution of silver nanoparticles was synthesized by the well known chemical reduction method ${ }^{1}$. In a typical experiment $\mathrm{AgNO}_{3}\left(1 \times 10^{-3} \mathrm{M}\right)$ solution was mixed with ice-chilled sodium borohydride $\left(2 \times 10^{-3} \mathrm{M}\right)$ solution in a 1:3 volume ratio with constant stirring. The resulting silver hydrosols exhibited a yellow colour.

Preparation of polyelectrolytes solutions: A stock solution of $1 \times 10^{-3} \mathrm{M} \mathrm{NaCl}$ was prepared using 18.2 Milli-Q water. This solution was used for preparing $1 \mathrm{mg} / \mathrm{mL}$ PSS, PDADMAC and PAH-FITC solutions.

Modification of quartz slides: The Quartz (CHEMGLASS, $25 \mathrm{~mm} \times 25 \mathrm{~mm}$ ) slides were rinsed with acetone followed by sonication in concentrated $\mathrm{H}_{2} \mathrm{SO}_{4}$ for $0.5 \mathrm{~h}$. After washing copiously with $18.2 \mathrm{M} \Omega$ Mili-Q water and then cleaned slides were heated in piranha solution consisted of 5:1:1 by volume
$\mathrm{H}_{2} \mathrm{O}: \mathrm{NH}_{3}: \mathrm{H}_{2} \mathrm{O}_{2}$ at $70{ }^{\circ} \mathrm{C}$ for $10 \mathrm{~min}$. These slides were thoroughly rinsed with water and subsequently dried with $\mathrm{N}_{2}$ gas. The slides were then immersed in a freshly prepared PDADMAC solution for $15 \mathrm{~min}$. All the slides were subjected to drying in a stream of $\mathrm{N}_{2}$ gas prior to the deposition of metal nanoparticles.

Engineering 2D assembly of nanoparticles on modified quartz substrate: The substrates prepared in previous steps were dipped into a colloidal solution of silver nanoparticles for $24 \mathrm{~h}$ followed by rinsing with 18.2 Milli-Q water for $3 \mathrm{~min}$. Deposition of the nanoparticles on the substrates was monitored by the surface plasmon resonance (SPR) absorption spectra of those substrates using CARY 3 UV-visible spectrophotometer.

Deposition of polyelectrolytes multilayer: The polyelectrolytes layers were deposited by successively dipping the substrate (with or without a monolayer of silver nanoparticles) into the solution containing negatively charged polyelectrolyte PSS for $15 \mathrm{~min}$, after which the substrates were rinsed with 18.2 Milli-Q water for $2 \mathrm{~min}$ and then dipped into the oppositely charged polyelectrolyte, PDADMAC, solution for another $15 \mathrm{~min}$. This process was repeated until the desired number of layers was achieved. The growth of the layers was monitored by UV-visible spectra of polyelectrolyte multilayers after five bilayers of polyelectrolyte (successive deposition of PSS/ PDADMAC is referred to as one bilayer throughout this report) had been deposited. The absorption intensity of PSS at $227 \mathrm{~nm}$ was used as a reference signal for monitoring the layer growth. A clean quartz slide treated with piranha solution was used as a reference for the spectral measurements. All the UVvisible spectra were processed by GRAMS/AI software.

Deposition of dyes on the substrate: Dyes (Rh6G and FITC) were deposited on the top of polyelectrolyte multilayers with negatively charged PSS being the top layer using the method described by Wang et al. ${ }^{18}$. Briefly, the substrates (with or without nanoparticles) containing different number of polyelectrolyte bilayers were dipped into the solutions of Rh6G $\left(\mathrm{pH} 2.5,1 \times 10^{-3} \mathrm{M}\right)$ and PAH-FITC $(\mathrm{pH} 4,1 \mathrm{mg} / \mathrm{mL})$ for 20 min. However, three layers of PAH-FITC were deposited onto the polyelectrolyte multilayers instead of one as in the case of Rh6G. This was done by deposition of PAH-FITC on the top PSS layer and further deposition of two additional PSS/PAHFITC bilayers. After immersion, the substrates were rinsed thoroughly with 18.2 Milli-Q water for 2 min followed by drying in a stream of nitrogen.

Raman and fluorescence analysis: Raman and fluorescence measurements were performed using a single grating monochromator (Jobin Yvon, focal length $640 \mathrm{~nm}$ ) equipped with a liquid nitrogen cooled CCD camera (Princeton Instruments) and a notch filter to remove the excitation wavelength. Argon ion laser system (Coherent), operating at $488 \mathrm{~nm}$ excitation wavelength with an output power of $0.48 \mathrm{~mW}$ was used for excitation. The spectral resolution of the instrument was $4 \mathrm{~cm}^{-1}$. The accumulation time was $60 \mathrm{~s}$. The Raman spectra were collected by WinSpec/32 software and further processed by GRAMS/AI software.

Topographic measurements: The topographic image of the supported metal nanoparticles assembly was taken on a multimode Nanoscope III (Digital instruments, Santa Barbara, 
CA) or Ntegra (NTMDT, Russia) atomic force microscope (AFM) in the contact mode in air. A J scanner with maximal scan area of $120 \mu^{2}$ (Nanoscope) or $100 \mu \mathrm{m} \times 100 \mu \mathrm{m}$ (Ntegra) and $200 \mu \mathrm{m}$ long soft cantilevers with integrated pyramidal silicon nitride tips having spring constant of $60 \mathrm{mN} /$ $\mathrm{m}$ were used for all topographic measurements. The imaging force was approximately $2-4 \mathrm{nN}$ and the scan rate was typically $0.5 \mathrm{~Hz}$.

\section{RESULTS AND DISCUSSION}

Characterization of 2D assembly of silver nanoparticles: 2D assembly of silver nanoparticles was formed by dipping quartz substrate, functionalized with PDADMAC, into the colloidal solution of silver nanoparticles for $24 \mathrm{~h}$. Adhesion of silver nanoparticles on the substrate is governed by the electrostatic attraction between the negatively charged silver nanoparticles and positively charged PDADMAC ${ }^{1,2,16}$. Formation of silver nanoparticles monolayer was confirmed by AFM topographic image of the substrate (Fig. 1A). The AFM image shows a monolayer of spherodial shaped silver nanoparticles having diameter $20 \mathrm{~nm}$ with some aggregates. The surface plasmon resonance (SPR) absorption spectra of the supported nanoparticle monolayer, shown as solid line in Fig. 1B, exhibits a characteristic peak centered at $400 \mathrm{~nm}$ corresponding to resonance excitation of localized surface plasmon of $20 \mathrm{~nm}$ nanoparticles. However, for the colloidal nanoparticles, the peak was noticed at $391 \mathrm{~nm}$ (shown by dotted line in Fig. 1B). This red shifting of SPR absorption maxima by $9 \mathrm{~nm}$ is attributed to the change of the surrounding dielectric environment ${ }^{19}$.
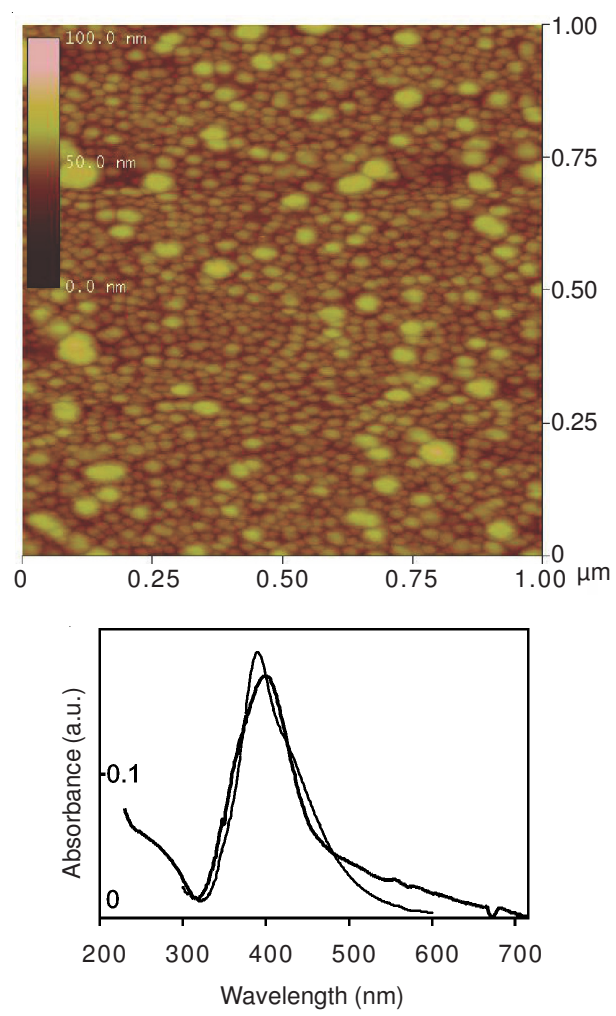

Fig. 1. (A) AFM topography image of a substrate containing a monolayer of silver nanoparticles deposited on modified quartz slide, (B) the corresponding surface plasmon resonance absorption spectra of the silver nanoparticle monolayer (solid line) and the spectrum of the colloidal nanoparticles solution (dotted line)
Growth of polyelectrolyte multilayer films: We prepared two series of substrates: one series contained Rh6G (SchemeIA) and another contained PAH-FITC (Scheme-IB). In each series variable number $(n=1,5,10,20$ and 30$)$ of polyelectrolytes bilayers (PSS/PDADMAC) was deposited on five different substrates each containing a monolayer of silver nanoparticles. Electrostatic interaction between oppositely charged polyelectrolytes and subsequent charge reversal are the driving forces for polyelectrolyte film growth. The evolution of the polyelectrolyte film thickness was monitored by measuring the absorption intensity of the phenyl group in PSS at $227 \mathrm{~nm}$ (Figs. 2A and 4A). Plots of PSS absorption intensity as a function of number of bilayers on both series show a linear film growth of polyelectrolyte multilayer films. The growth of films thickness has been presented in Fig. 2B for Rh6G and Fig. 4B for PAH-FITC. Such linear dependence of thickness reveals regular deposition of polyelectrolyte in each deposition cycle which is crutial for distance controlled experiments ${ }^{16}$.

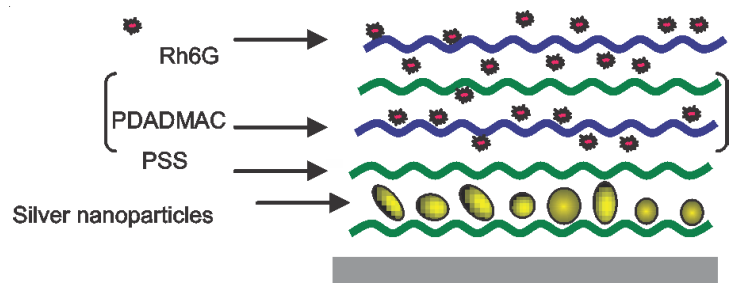

(A)

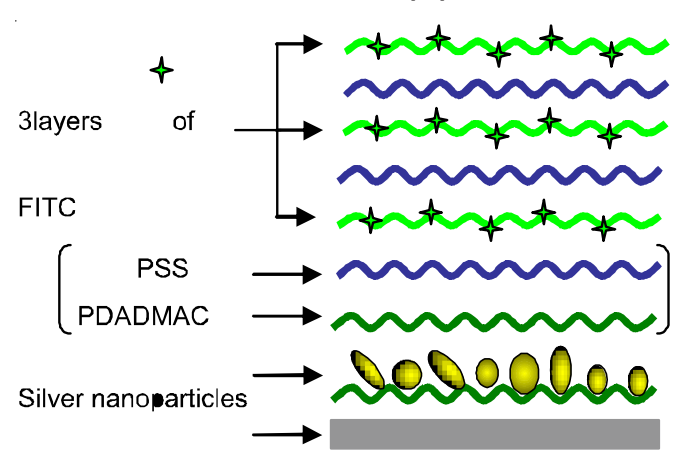

(B)

Scheme-I: Schematic representations of typical metal nanoparticlepolyelectrolyte nanocomposities containing Rh6G and FITC. Scheme-I(A) shows a monolayer of silver nanoparticles having different number of PSS/PDADMAC bilayers with Rh6G on the top. Diffusion of Rh6G through polyelectrolyte film is a characteristic feature of this substrate. Scheme-I(B) shows a monolayer of silver nanoparticles having different number of polyelectrolyte bilayers with three layers of PSS/PAH-FITC on the top. FITC being bonded to PAH backbone can not diffuse through polyelectrolyte film in this substrate

Deposition of Rh6G and PAH-FITC on polyelectrolyte films: Dyes were deposited on the top of each polyelectrolyte multilayer films as described. In acidic pH, both Rh6G and PAH-FITC exist in cationic form because of the protonation of amino groups. Electrostatic attraction between cationic dyes and anionic PSS layers of polyelectrolyte films is the driving force for the adsorption process. To achieve a uniform distribution of dyes on the polyelectrolyte film surface, all the substrates were subjected to the same deposition conditions i.e.; $\mathrm{pH}$ and concentration of the dye solutions, incubation time, rinsing and drying process. 

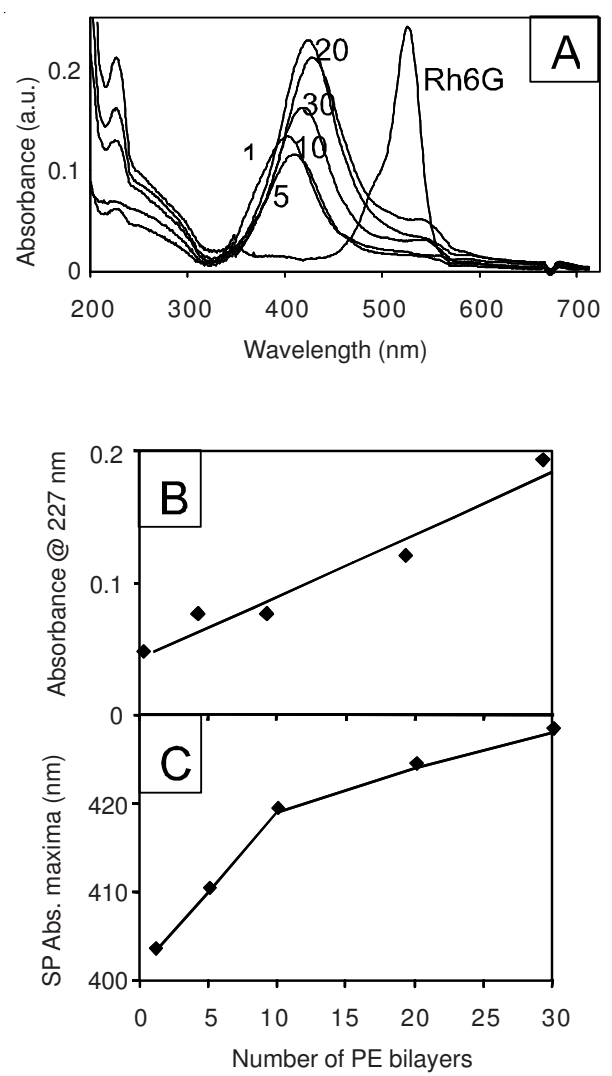

Fig. 2. (A) UV-visible absorption spectra of the substrates containing nanoparticles, a variable number $(1,5,10,20$ and 30$)$ of polyelectrolyte bilayers (PSS/PDADMAC) and Rh6G. Solution phase spectrum of $\mathrm{Rh} 6 \mathrm{G}\left(1 \times 10^{-6} \mathrm{M}, \mathrm{pH} c a\right.$. 2.5) is shown to track the position of Rh6G absorption signal of the substrates. (B) Absorbance at $227 \mathrm{~nm}$ was used to monitor the growth of polyelectrolyte film. (C) Position of surface plasmon (SP) absorption maxima shifts as the number of polyelectrolyte bilayers increases

Distribution of fluorophores onto the polyelectrolyte film: Reliable investigations of the lateral and axial dependence of Raman and fluorescence enhancement depend on the ability to position the probing molecules at a well defined distance from the nanoparticles surface. In this work absorption signal of Rh6G and FITC was used to confirm the homogeneity of the fluorophores distribution.

Fig. 2A shows the UV-visible absorption spectra of the substrates containing Rh6G as well as its solution phase spectrum. The formation of the polyelectrolyte film on nanoparticles monolayer was monitored by PSS absorbance intensity at $227 \mathrm{~nm}$ (Fig. 2B) as well as by the progressive change in the position of the surface plasmon absorption band (Fig. 2C), which is known to be sensitive to the near-field change of dielectric constant $\mathrm{t}^{6,20}$. In aqueous solution Rh6G shows absorption peak at $520 \mathrm{~nm}^{21}$. It can be seen from Fig. 2A that as the number of polyelectrolyte bilayers increases, the intensity of the absorption peak around $520 \mathrm{~nm}$ and consequently the amount of Rh6G increased as well. When plotted (Fig. 3) a linear dependence of the amount of Rh6G on the number of polyelectrolyte bilayers is obtained. This implies that dye content of the substrates increases with increasing thickness of bilayers. Since the dye deposition conditions throughout the experiment, the only possibility is the diffusion of Rh6G molecules through the polyelectrolyte film matrix with increasing layer thickness.

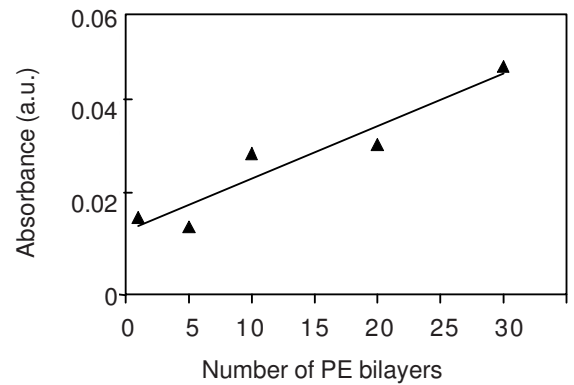

Fig. 3. Increase in the amount of Rh6G deposited onto the substrates with increasing number $(1,5,10,20$ and 30$)$ of polyelectrolyte bilayers (PSS/PDADMAC) is monitored by using the absorption band of Rh6G at $520 \mathrm{~nm}$

Same experiment was carried out with PAH-FITC, a polyelectrolyte loaded with fluorophore (Fig. 4). In aqueous solution FITC ( $\mathrm{pH} \mathrm{4)}$ shows an absorption peak at 496 nm, Fig. 4A. Three rather than one layers of PAH-FITC were deposited on the top of nanocomposite substrates (SchemeIB) in order to simplify the detection. However, no detectable signal from the adsorbed fluorophores was noticed around 496 $\mathrm{nm}$ even for 30 polyelectrolyte bilayers. Film thickness behavoiur (Fig. 4B) and surface plasmon resonance position (Fig. 4C) were similar to that of Fig. 2 experiment.
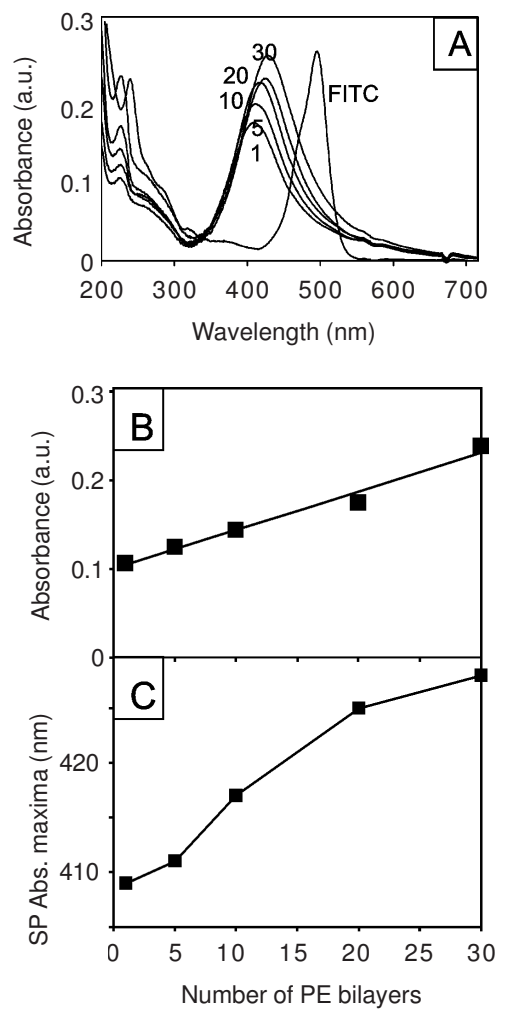

Fig. 4. (A) UV-VIS absorption spectra of the substrates containing nanoparticles, a variable number (1, 5, 10, 20 and 30) of polyelectrolyte bilayers (PSS/PDADMAC) and PAH-FITC. Solution phase spectrum of PAH-FITC $\left(1 \times 10^{-6} \mathrm{M}, \mathrm{pH}\right.$ ca. 4$)$ is shown to track the position of PAH_FITC absorption signal of the substrates. (B) Absorbance at $227 \mathrm{~nm}$ was used to monitor the growth of polyelectrolyte film. (C) Position of surface plasmon (SP) absorption maxima shifts as the number of polyelectrolyte bilayers increases

To detect the absorption signal of FITC, the number of PAH-FITC layers was increased. However, in this case 
PDADMAC was replaced by PAH-FITC. To eliminate the SPR absorption signal of nanoparticles, polyelectrolytes layers were deposited without any nanoparticles. The UV-VIS absorption spectra of these substrates are shown in Fig. 5. The PSS/PAHFITC film growth also follows a linear fashion (Fig. 5, inset bottom). We noticed a detectable absorption signal of FITC only after 10 layers of FITC had been deposited and observed a linear increase of the absorbance with the number of PAHFITC layers (Fig. 5).

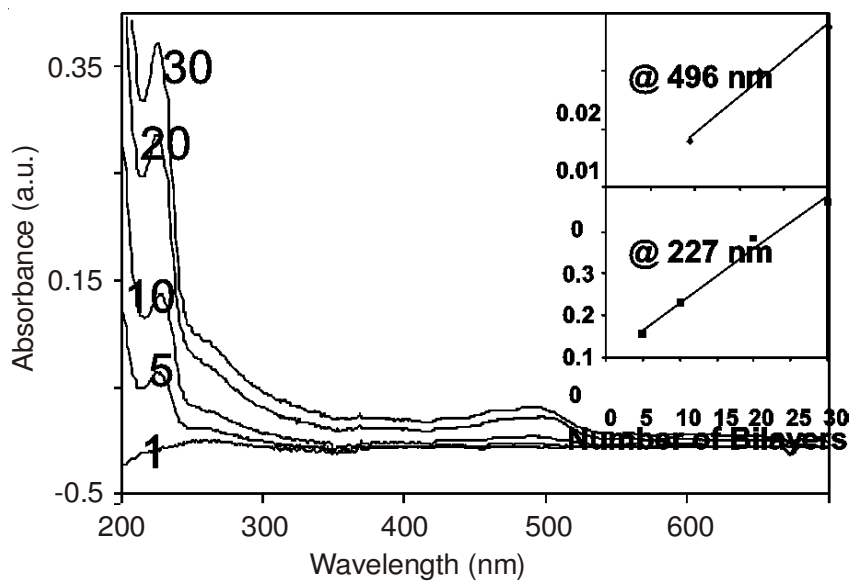

Fig. 5. UV-VIS absorption spectra of the substrates without nanoparticle containing variable number $(1,5,10,20$ and 30) of polyelectrolyte bilayers (PSS/PAH-FITC). PSS absorption at $227 \mathrm{~nm}$ was observed only after the deposition of 5 bilayers and a detectable FITC absorption (at $496 \mathrm{~nm}$ ) signal was seen after deposition of 10 bilayers. Growth of PSS/PAH-FITC polyelectrolyte film (absorption@227 $\mathrm{nm}$ ) and variation of FITC absorption intensities (absorption @ 496 $\mathrm{nm}$ ) with increasing number of polyelectrolyte bilayers are shown

These results suggest that unlike Rh6G, polyelectrolyte bound fluorophore FITC does not diffuse through the polyelectrolyte film matrix.

Effect of local environment on surface plasmon resonance absorption: It is known that a change in the dielectric medium around the nanoparticles brings about a red shift in plasmon resonance absorption ${ }^{6}$. In this present study, dielectric medium was altered by increasing the thickness of the film. The effect of local environment on the position of plasmon resonance absorption is presented in Fig. 2C for Rh6G series and $4 \mathrm{C}$ for PAH-FITC series. Plasmon resonance absorption peak was noticed to increasing steadily up to 20 bilayers (corres-ponds to $20 \mathrm{~nm}$ ). We assume that local electromagnetic field associated with $20 \mathrm{~nm}$ metal nanoparticles extends to this distance. Within the region confined by the limiting distance from the nanoparticle surface, attendance of dielectric materials reduces the strength of local electromagnetic field around the nanoparticle which in turn lowers the frequency of plasmon oscillation $^{6}$. Once the dielectric function within the limiting region is established further introduction of polyelectrolyte layers does not bring any significant change in plasmon resonance shift ${ }^{6,22}$.

Concentration correction to surface enhanced Raman scattering and surface enhanced fluorescence of the nanocomposities: It is important to normalize Raman and fluorescence intensities of the substrates containing Rh6G to account for the variation of dye concentration in the substrates as a result of diffusion. For concentration correction, we employed a control substrate which was a modified quartz slide containing Rh6G (without polyelectrolyte and nanoparticle). We introduced a term correction factor for normalizing Raman and fluorescence intensities of the substrates. Correction factor (CF) is defined as the ratio of Rh6G absorption intensity in the nanocomposite substrate $\left({ }^{\mathrm{Rh} 6 \mathrm{G}} \mathrm{I}_{\mathrm{NC}}\right)$ to that of in the control substrate $\left({ }^{\mathrm{Rh} 6 \mathrm{G}} \mathrm{I}_{\mathrm{CON}}\right)$ i.e.; C.F. $={ }^{\mathrm{Rh} 6 \mathrm{G}} \mathrm{I}_{\mathrm{NC}} /{ }^{\mathrm{Rh} 6 \mathrm{G}} \mathrm{I}_{\mathrm{CON}}$. Finally, the Raman and fluorescence intensities of individual substrates were divided by their corresponding correction factors to obtain the normalized Raman and fluorescence intensity.

Enhancement factor calculation: Magnitude of optical signal enhancement in SERS or SEF is estimated by enhancement factor. Enhancement factor (EF) is defined by the following relation $\mathrm{EF}=\mathrm{I}_{\mathrm{Ag}} / \mathrm{I}_{0}=\mathrm{I}_{\mathrm{SERS}} / \mathrm{I}_{\mathrm{RS}}\left(\right.$ or $\left.\mathrm{I}_{\mathrm{SEF}} / \mathrm{I}_{\mathrm{F}}\right)$ where $\mathrm{I}_{\mathrm{Ag}}$ and $I_{0}$ are the intensities of Raman (or fluorescence) signal of dye molecules adsorbed on silver nanoparticles substrates and that of on substrates without silver nanoparticles, respectively. $\mathrm{I}_{\mathrm{SERS}}$ (or $\mathrm{I}_{\mathrm{SEF}}$ ) and $\mathrm{I}_{\mathrm{RS}}$ (or $\mathrm{I}_{\mathrm{F}}$ ) are the Raman (or fluorescence) intensities of the dyes under SERS (or SEF) and non-SERS (non-SEF) conditions, respectively.

Surface enhanced Raman scattering activities of nanocomposities: Metal nanoparticle has long been known to enhance the Raman scattering of the molecules adsorbed on their surface as a result of intense EM field, induced by external EM radiation, around the nanoparticles. The intensity of EM induced is reported to decrease away from the surface ${ }^{3,23-29}$. To investigate distance dependent characteristics of SERS, we employed the aforementioned substrates containing silver nanoparticles and dyes (Rh6G and PAH-FITC) and the distance between them was created by monolayer with variable number of polyelectrolyte (PDADMAC/PSS) bilayers as spacer between the silver nanoparticle monolayer and the dyes. A bare slide containing dye adsorbed on if used as a control for estimating SERS enhancement factor.

Fig. 6 shows the SERS spectra (with fluorescence background) of the substrates containing Rh6G. It is evident that SERS spectrum of the control substrates containing Rh6G monolayer (indicated by ' 0 ') is very week and cannot be resolved under the experimental conditions used. However, one can observe a weak background due to the fluorescence of Rh6G. The addition of the nanoparticles to the substrates leads to a dramatic increase in the Raman scattering. The bands observed at 1364, 1513, 1581 and $1665 \mathrm{~cm}^{-1}$, assigned to aromatic $\mathrm{C}-\mathrm{C}$ stretching vibrations of $\mathrm{Rh} 6 \mathrm{G}$ are the characteristics of Rh6G SERS peaks ${ }^{29,30}$. Intensities of the band around $1665 \mathrm{~cm}^{-1}$ for different substrates were measured and normalized for concentration correction as described earlier. SERS enhancement factors of different substrates were estimated from these normalized intensities. Maximum SERS enhancement was observed for the substrate containing Rh6G layer separated by a single polyelectrolyte bilayer from the nanoparticle monolayer. Moreover, SERS intensity was found to decrease with increasing number of polyelectrolyte bilayers (Fig. 7). The enhancement of SERS scattering is caused by induced local EM field around ${ }^{31-34}$. At this vicinity of the nanoparticles surface intensity of EM field is highest and decrease 


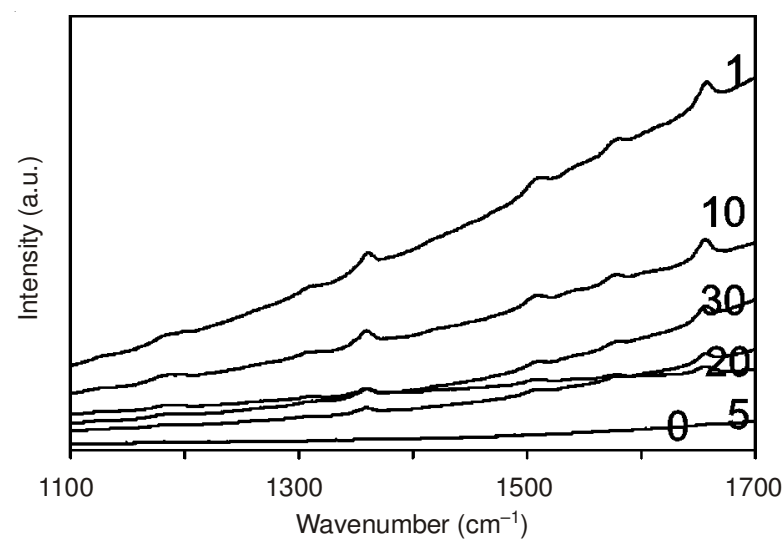

Fig. 6. SERS spectra (with fluorescence background) for substrates containing a monolayer of silver nanoparticles covered with variable number (1, 5, 10, 20 and 30) of polyelectrolyte bilayers (PSS/ PDADMAC) and Rh6G. ' 0 ' refers to a substrate used as control with Rh6G deposited on bare (without silver nanoparticle and polyelectrolyte bilayers) quartz slide

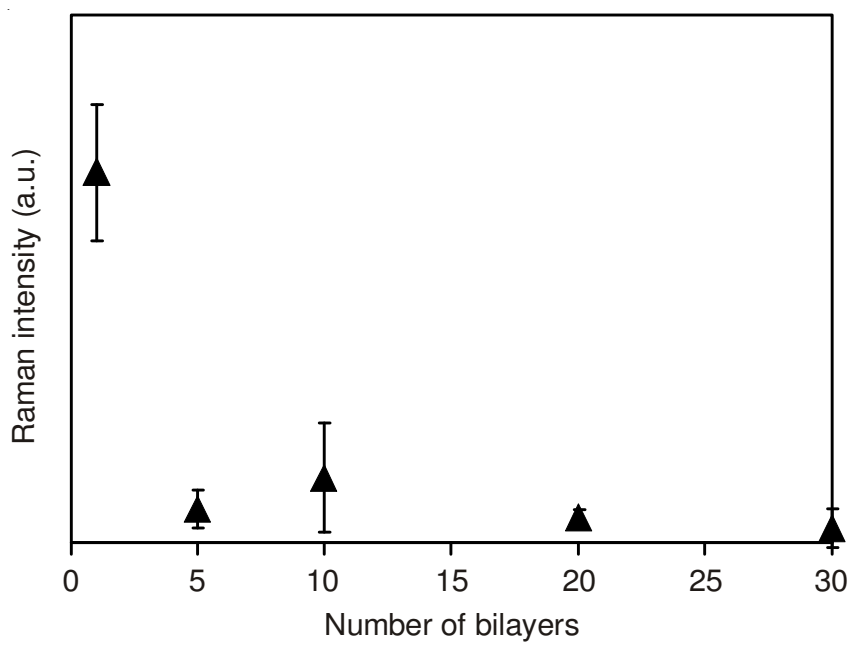

Fig. 7. Distance dependence of the Rh6G SERS signal measured by using the intensity of the band at $1665 \mathrm{~cm}^{-1}$ as a function of the number of bilayers from the Fig. 6 spectra. Intensity was adjusted by using the Fig. 3 data for the amount of Rh6G deposited on the substrates

away from it. Therefore, enhancement of SERS decreases with increasing number of polyelectrolyte bilayers $(5,10,20$ and 30). Such distance dependent behavoiur of SERS has been previously reported ${ }^{35,36}$. No detectable SERS signal of FITC was detected as the SERS peaks were engulfed by its huge fluorescence background.

Surface enhanced fluorescence activities of nanocomposities: Similar to SERS, fluorescence emission of a nearby dipole is modified by metal nanoparticles. However, both the fluorescence enhancement and quenching by metal nanoparticles have been reported in the literature ${ }^{37}$. In this present study, both series of substrates containing Rh6G and FITC exhibited fluorescence enhancement effect. Fluorescence emission spectra of the substrates containing Rh6G and FITC with different number of polyelectrolyte bilayers (1, 5, 10, 20 and 30) are presented in Fig. 6 (as a background of Raman signal) and 8, respectively. Fluorescence enhancement factors were calculated as described earlier. We found a 14 fold enhancement for Rh6G and a 2 fold enhancement for FITC. Investigation of distance dependent fluorescence of Rh6G reveals that maximum enhancement occurs at a separation distance corresponding to one polyelectrolyte bilayer $(1 \mathrm{~nm})$ and then decreases with increasing number of bilayers (Fig. 9). Surprisingly, we did not observe quenching at the short distances reported in literature $^{38,39}$. The observed decrease in the fluorescence EF with higher number of bilayers (5-30) might be due to the formation of nonfluorescent aggregates as a result of Rh6G diffusion through the polyelectrolyte matrix as described earlier ${ }^{20}$. Distance dependent fluorescence study of PAH-FITC (Fig. 9) shows that fluorescence intensity increases with increasing number of bilayer. The observed fluorescence enhancement is the result of two phenomena: Firstly, the static quenching of FITC fluorescence by metal nanoparticles through nonradiative pathways and secondly, increased radiative decay rates induced by the enhanced EM field around the nanoparticles. Since quenching decays from the surface faster than the local EM field therefore, fluorescence intensity increases as the distance between the nanoparticles and the dye increases ${ }^{6}$. Compared to Rh6G, PAH-FITC exhibits an increasing trend of fluorescence enhancement with increasing number of bilayers. It is likely that FITC molecules are bonded to $\mathrm{PAH}$ backbone which retards the diffusion of molecules into the polyelectrolyte matrix which prevents the formation of any nonfluorescent aggregates. Another important feature is the lower fluorescence EF compares to the Rh6G which might be cause by the self quenching of the fluorescence by the FITC $^{40,41}$.

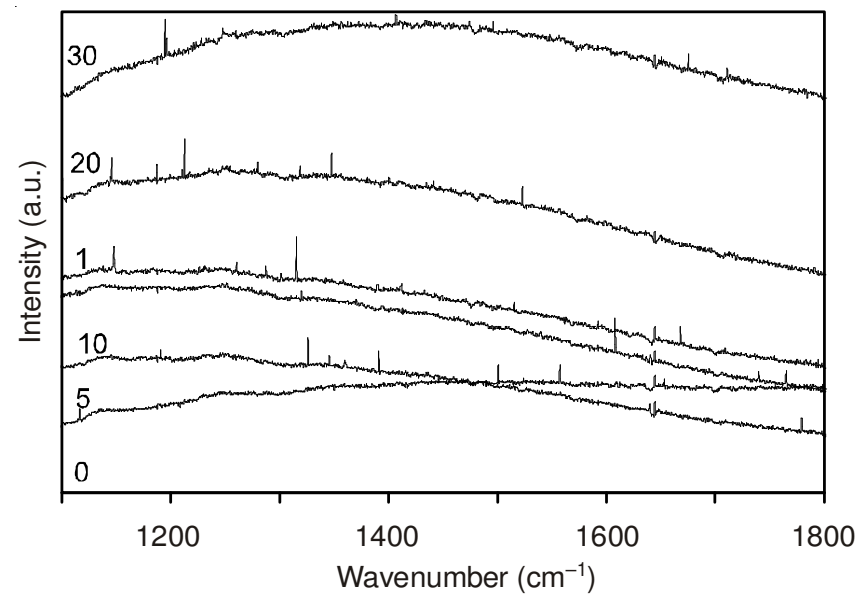

Fig. 8. Fluorescence emission spectra of the substrates containing silver nanoparticle, a variable number $(1,5,10,20$ and 30) of polyelectrolyte bilayers (PSS/PDADMAC) with three PSS/PAHFITC bilayers adsorbed on the top. '0' refers to a substrate used as control having PAH-FITC deposited on bare (without silver nanoparticle and polyelectrolyte bilayers) quartz slide

\section{Conclusion}

We fabricated silver nanoparticle-polyelectrolyte nanocomposite substrates having varying thickness and deposited two different dyes, Rh6G and PAH-FITC, on the top of the polyelectrolytes film by layer by layer self assembly process to study the distance dependent nature of SERS and SEF exhibited by these nanocomposite substrates. We found a significant diffusion of Rh6G molecules through polyelectrolyte film matrix, which subsequently formed nonfluorescent aggregates. On the contrary, PAH-FITC did not exhibit any 


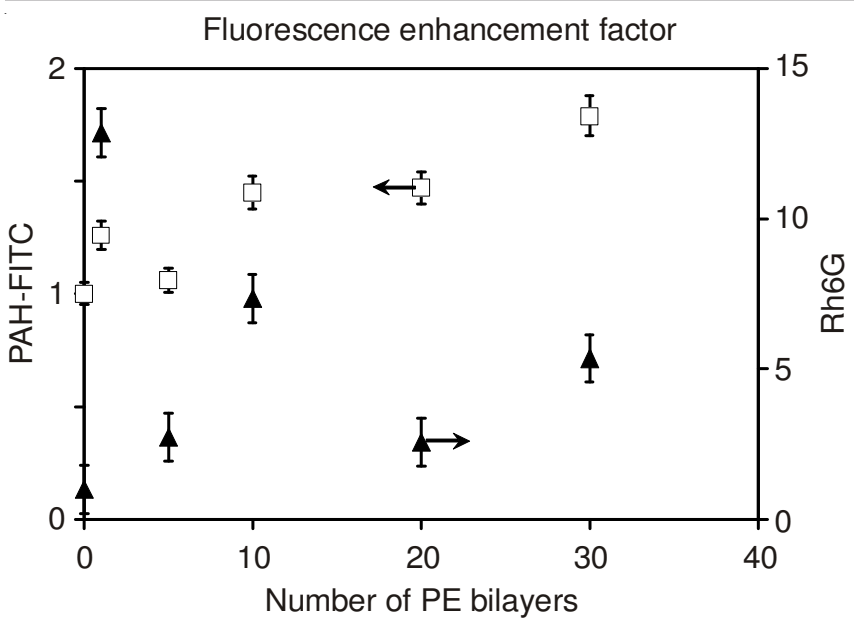

Fig. 9. Distance dependent fluorescence enhancement of Rh6G (triangle) and FITC (rectangle) deposited on substrates containing silver and with increasing number $(1,5,10,20$ and 30) of polyelectrolyte bilayers (PSS/PDADMAC). '0' refers to the substrates used as controls having Rh6G or PAH-FITC deposited on bare (without nanoparticle and polyelectrolyte bilayers) quartz slides. The enhancement factors for Rh6G and FITC were estimated from the emission spectra presented in Figs. 6 and 8, respectively

diffusion as it is bound to the polyelectrolyte chain. Both the substrates containing Rh6G and PAH-FITC exhibited SERS and SEF activities. Distance dependent fluorescence studies showed a maximum of 14-fold enhancement of Rh6G when it is separated from the nanoparticles by one polyelectrolyte bilayer (PSS/PDADMAC). A 2-fold fluorescence enhancement of PAH-FITC was observed when it was separated from the nanoparticles by 30 polyelectrolyte bilayers. We observed maximum Raman enhancement at a separation distance of one polyelectrolyte bilayer for the case of Rh6G. Finally, we conclude that when performing Raman and fluorescence based experiments it is best to employ immobilized dyes, such as PAH-FITC, rather than mobile dyes, Rh6G, to avoid complexity arising from diffusion of dye molecules through the spacer film matrix.

\section{ACKNOWLEDGEMENTS}

The authors thank Graham Galway for his assistance in this research. Financial support was provided by NSERC, CFI and Carleton University.

\section{REFERENCES}

1. A. N.Shipway, E. Katz and I. Willmer, Chem. Phys. Chem., 1, 18 (2000).

2. F. Caruso, M. Spasova, V. Salgueiriño-Maceira and L.M. Liz-Marzán, Adv. Mater., 13, 1090 (2001)

3. A. Campion and K. Patanjali, Chem. Soc. Rev., 27, 241 (1998).

4. X. Huang, I.H. El-Sayed, W. Qian and M.A. El-Sayed, J. Am. Chem. Soc., 128, 2115 (2006)
5. E. Ghiamati, M. Lashkari and M. Hasheminia, Asian J. Chem., 25, 1361 (2013).

6. D.D. Evanoff Jr., R.L.White and G. Chumanov, J. Phys. Chem. B, 108, 1522 (2004).

7. P.K. Jain, W. Huang and M.A. El-Sayed, Nano Lett., 7, 2080 (2007).

8. K.F. Domke, D. Zhang and B. Pettinger, J. Phys. Chem. C, 111, 8611 (2007).

9. J. Zhang, Y. Fu, M.H. Chowdhury and J.R. Lakowicz, Nano. Lett., 7, 2101 (2007).

10. A. Ianoul and A. Bergeron, Langmuir, 22, 10217 (2006).

11. K. Ray, R. Badugu and J.R. Lakowicz, Chem. Mater., 19, 5902 (2007).

12. S. Thomas, C. Lobmaier and F. Pittner, Proc. SPIE, 129, 2976 (1997).

13. G. Chumanov, K. Sokolov, B.W. Gregory and T.M. Cotton, J. Phys. Chem., 99, 9466 (1995).

14. G. Decher, Science, 277, 1232 (1997).

15. T.R. Farhat and P.T. Hammond, Adv. Funct. Mater., 16, 433 (2006).

16. P.T. Hammond, Curr. Opin. Colloid Interf. Sci., 4, 430 (2000).

17. G. Decher, Comprehensive Supramolecular Chemistry: Templeting, Layered Nanoarchitectures via Directed Assembly of Anionic and Cationic Molecules, Pergamon Press, Oxford, Vol. 9, p. 507 (1996).

18. Y. Wang and C. Hu, Thin Solid Films, 476, 84 (2005).

19. J. Schmitt, P. Machtle, D. Eck, H. Mohwald and C.A. Helm, Langmuir, 15, 3256 (1999).

20. J. Zhao, L. Jensen, J. Sung, S. Zou, G.C. Schatz and R.P. van Duyne, J. Am. Chem. Soc., 129, 7647 (2007).

21. R. Reisfeld, R. Zusman, Y. Cohen and M. Eyal, Chem. Phys. Lett., 147, 142 (1988).

22. L.J. Sherry, S.H. Chang, G.C. Schatz, R.P. Van Duyne, B.J. Wiley and Y. Xia, Nano. Lett., 5, 2034 (2005).

23. T.R. Jensen, M.L. Duval, K.L. Kelly, A.A. Lazarides, G.C. Schatz and R.P. Van Duyne, J. Phys. Chem. B, 103, 9846 (1999).

24. M. Futamata, Y. Maruyama and M. Ishikawa, Vibrat. Spectrosc., 30, 17 (2002).

25. S. Dong and X. Zou, J. Phys. Chem. B, 110, 21545 (2006).

26. K. Kwon, K.Y. Lee, Y.W. Lee, M. Kim, J. Heo, S.J. Ahn and S.W. Han, J. Phys. Chem. C, 111, 1161 (2007).

27. M. Ringler, T.A. Klar, A. Schwemer, A.S. Susha, J. Stehr, G. Raschke, S. Funk, M. Borowski, A. Nichtl, K. Kurzinger, R.T. Phillips and J. Feldmann, Nano Lett., 7, 2753 (2007).

28. E.C. Le Ru, E. Blackie, M. Meyer and P.G. Etchegoin, J. Phys. Chem. C, 111, 13794 (2007).

29. D. Pristinski, S. Tan, M. Erol, H. Du and S. Sukhishvili, J. Raman Spectrosc., 37, 762 (2006).

30. P. Hildebrandt and M. Stockburger, J. Phys. Chem., 88, 5935 (1984).

31. J. Zhang, X. Li, X. Sun and Y. Li, J. Phys. Chem., 109, 12544 (2005).

32. A.F. McCabe, D. Graham, D. McKeown and W.E. Smith, J. Raman Spectrosc., 36, 45 (2005).

33. E.C. Le Ru and P.G. Etchegoin, Chem. Phys. Lett., 396, 393 (2004).

34. P. Kambhampati, C.M. Child, M.C. Foster and A.J. Campion, J. Chem. Phys., 108, 5013 (1998).

35. G. Compagnini, C. Galati and S. Pignataro, Phys. Chem. Chem. Phys., 1, 2351 (1999).

36. Q. Ye, J. Fang and L. Sun, J. Phys. Chem. B, 101, 8221 (1997).

37. Y. Fu and J.R. Lakowicz, J. Phys. Chem. B, 110, 22557 (2006).

38. J.R. Lakowicz, Anal. Biochem., 298, 1 (2001).

39. J.R. Lakowicz, Anal. Biochem., 337, 171 (2005).

40. C. Deka, B.E. Lehnert, N.M. Lehnert, G.M. Jones, L.A. Sklar and J.A. Steinkamp, Cytometry, 25, 271 (1996).

41. J.R. Lakowicz, J. Malicka, S.D'Auria and I. Gryczynski, Anal. Biochem., 320, 13 (2003). 